\title{
ON THE STRUCTURE OF LOCALLY POTENTIALLY EQUIVALENT GALOIS REPRESENTATIONS
}

\author{
VIJAY M. PATANKAR AND C. S. RAJAN
}

\begin{abstract}
Suppose $\rho_{1}, \rho_{2}$ are two $\ell$-adic Galois representations of the absolute Galois group of a number field, such that the algebraic monodromy group of one of the representations is connected and the representations are locally potentially equivalent at a set of places of positive upper density. We classify such pairs of representations and show that up to twisting by some representation, it is given by a pair of representations one of which is trivial and the other abelian.

Consequently, assuming that the first representation has connected algebraic monodromy group, we obtain that the representations are potentially equivalent, provided one of the following conditions hold: (a) the first representation is absolutely irreducible; (b) the ranks of the algebraic monodromy groups are equal; (c) the algebraic monodromy group of the second representation is also connected and (d) the commutant of the image of the second representation remains the same upon restriction to subgroups of finite index of the Galois group.
\end{abstract}

\section{INTRODUCTION}

Two $n$-dimensional linear representations $\rho_{1}, \rho_{2}$ of a group $\Gamma$ are said to be potentially equivalent if they become isomorphic upon restriction to a subgroup of finite index in $\Gamma$. In this article, we are interested in proving potential equivalence of $\ell$ adic representations of the absolute Galois group of a global field, which are locally potentially equivalent at a sufficiently large number of places of the global field.

For a field $L$, denote by $G_{L}:=\operatorname{Gal}(\bar{L} / L)$ an absolute Galois group of $L$, where $\bar{L}$ denote a seperable algebraic closure of $L$. Let $K$ be a global field, i.e., a number field or the function field of a curve over a finite field. For a place $v$ of $K$ let $K_{v}$ denote the completion of $K$ at $v$. Choosing a place $w$ of $\bar{K}$ lying above $v$, allows us to identity $G_{K_{v}}$ with the decomposition subgroup $D_{w}$ of $G_{K}$. As $w$ varies this gives a conjugacy class of subgroups of $G_{K}$. Given a representation $\rho$ of $G_{K}$ as above, define the localization (or the local component) $\rho_{v}$ of $\rho$ at $v$, to be the representation of $G_{K_{v}}$ obtained by restricting $\rho$ to a decomposition subgroup. This is well defined upto isomorphism.

Let $F$ be a local field of characteristic zero and residue characteristic $\ell$ relatively prime to the characteristic of $K$. Suppose $\rho_{i}: G_{K} \rightarrow G L_{n}(F), i=1,2$ are two continuous semisimple $\ell$-adic representations of $G_{K}$, unramified outside a finite set

1991 Mathematics Subject Classification. Primary 11F80; Secondary 11R45.

Key words and phrases. Galois representations; Potential equivalence; Algebraic Chebotarev density theorem. 
$\Sigma_{\text {ram }}$ of places of $K$ containing the archimedean places of $K$. Let $T$ be a set of places of $K$. Define $\rho_{1}$ and $\rho_{2}$ to be locally potentially equivalent at $T$, if for each $v \in T$, the restrictions $\rho_{1, v}$ and $\rho_{2, v}$ to $G_{K_{v}}$ are potentially equivalent.

Given a continuous representation $\rho: G_{K} \rightarrow G L_{n}(F)$ and a place $v$ of $K$ where $\rho$ is unramified, let $\rho\left(\sigma_{v}\right)$ denote the Frobenius conjugacy class in the image group $G_{K} / \operatorname{Ker}(\rho) \simeq \rho\left(G_{K}\right) \subset G L_{n}(F)$. By an abuse of notation, we will also continue to denote by $\rho\left(\sigma_{v}\right)$ an element in the associated conjugacy class. Assume further that the elements $\rho_{1}\left(\sigma_{v}\right)$ and $\rho_{2}\left(\sigma_{v}\right)$ are semisimple (by Corollary 2.1, this property is satisfied for semisimple representations at a set of places of density one). The representations $\rho_{1}$ and $\rho_{2}$ are locally potentially equivalent at a prime $v$ not in $\Sigma_{\text {ram }}$, precisely when the eigenvalues of the Frobenius conjugacy classes $\rho_{1}\left(\sigma_{v}\right)$ and $\rho_{2}\left(\sigma_{v}\right)$ differ by roots of unity.

For a representation $\rho: G_{K} \rightarrow G L_{n}(F)$, let $G_{\rho}$ be the algebraic monodromy group attached to $\rho$ over $F$, i.e., the smallest algebraic subgroup $G_{\rho}$ of $G L_{n}$ defined over $F$ such that $\rho\left(G_{K}\right) \subset G_{\rho}(F)$. Denote by $G_{i}$ the algebraic monodromy groups associated to the representations $\rho_{i}$ for $i=1,2$.

In [Ra], it is proved that if the representations $\rho_{1}$ and $\rho_{2}$ are locally equivalent (in fact, enough to assume only that the character values agree evaluated on the Frobenius element) at a set of unramified places having positive upper density and the algebraic monodromy group of one of the representations is connected, then $\rho_{1}$ and $\rho_{2}$ are potentially equivalent. We recall the upper density of a set $S$ of finite places of $K$ is defined as:

$$
u d(S):=\limsup _{x \rightarrow \infty} \#\{v \in S \mid N v \leq x\} / \pi(x),
$$

where $\pi(x)$ is the number of finite places $v$ of $K$ with $N v \leq x$. Here $N v$ denotes the cardinality of the residue field $k_{v}$ of $K_{v}$.

Based on this result, we consider the following generalization: assume that the algebraic monodromy group of either $\rho_{1}$ or $\rho_{2}$ is connected and the set of places $v$ of $K$ where the localizations $\rho_{1, v}$ and $\rho_{2, v}$ are potentially equivalent has positive upper density. Then are $\rho_{1}$ and $\rho_{2}$ (globally) potentially equivalent?

One of the motivations to consider this question is to understand the distribution of Frobenius fields of elliptic curves. Let $A$ be an abelian variety defined over a number field $K$. The Galois group $G_{K}$ acts in a natural manner on $A(\bar{K})$. For a rational prime $\ell$, the Tate module $T_{\ell}(A):=\lim _{n} A\left[\ell^{n}\right]$ is the $G_{K}$-module obtained as a projective limit of the $G_{K}$-modules $A\left[\ell^{n}\right]$ of $\ell^{n}$-torsion points of $A$ over $\bar{K}$. Let $V_{\ell}(A)=T_{\ell}(A) \otimes_{\mathbb{Z}_{l}} \mathbb{Q}_{l}$. The Tate module is of rank $2 d$ over the ring of $\ell$-adic integers $\mathbb{Z}_{\ell}$, where $d$ is the dimension of $A$.

When $A$ is an elliptic curve defined over $K$, we have a continuous $\ell$-adic representation $\rho_{A, \ell}: G_{K} \rightarrow G L_{2}\left(\mathbb{Q}_{\ell}\right)$. In [KPR, the above question is answered in the affirmative for the representations of $G_{K}$ acting on the Tate module of non-CM elliptic curves. At an unramified finite place $v$ of $K$ for $A$, the Frobenius field of $A$ at $v$ is 
the subfield of algebraic numbers generated by the eigenvalues of the Frobenius at $v$. As an application, it is shown that if the set of places of a number field $K$ at which the Frobenius fields of two non-CM elliptic curves defined over $K$ are isomorphic has positive upper density, then the curves are isogenous over a finite extension of $K$.

More generally, one can try to understand the multiplicative identities satisfied by the eigenvalues of a pair of Galois representations, say the natural representations on the Tate module attached to a pair of abelian varieties. We hope that the method outlined out here, might shed some light on the questions raised in ([Ko]).

Coming back to the question raised above, it was claimed in ([PR]) that if the algebraic monodromy group of one of the representations is connected and the upper density of the set $T$ of places of $K$ at which $\rho_{1}$ and $\rho_{2}$ are locally potentially equivalent is positive, then the representations $\rho_{1}$ and $\rho_{2}$ are potentially equivalent. It was pointed out by J.-P. Serre that this claim is wrong. The proof given in ([PR]) however goes through if we assume that the density of $T$ is one.

The problem was that in the course of the proof (see Remark 2.2), the following assertion was made: let $G$ be a reductive algebraic group, and let $m$ be a number divisible by the exponent of the group $G / G^{0}$. Then for any connected component $G^{\phi}$ of $G$, the $m$-th power map $G^{\phi} \rightarrow G^{0}$ is surjective, where $G^{0}$ is the connected component of identity in $G$.

Example 1.1. Serre gave the following counter-example: Let $G$ be the normalizer of the diagonal torus in SL(2). The square map sends the non-identity component to scalar matrices $( \pm \mathrm{Id})$ and is not surjective. In terms of representations, let $p_{1}$ denote the trivial two dimensional representation and $p_{2}$ the natural two dimensional representation of $G$. The characteristic polynomials of both these representations are equal evaluated at the fourth power of elements of the non identity connected component of $G$.

Example 1.2. Based on the above example, an arithmetical counterexample can be given to the claim made in $[\mathrm{PR}]$. Let $L=\mathbb{Q}(i)$ be the field of Gaussian numbers, and fix an embedding of $L$ into $\mathbb{C}$. Let $\ell$ be a rational prime that splits completely in $L$, and choose a prime $w$ of $L$ dividing $\ell$. Associated to an elliptic curve $E$ with complex multiplication by $\mathbb{Z}[i]$, there exists, by the theory of complex multiplication, a continuous character $\psi: G_{L} \rightarrow L_{w}^{*}$ with the following property: at a finite place $v$ of $L$ with residue field characteristic coprime to $\ell$ and unramified for $E$, the Frobenius element $\pi_{v}:=\psi\left(\sigma_{v}\right)$ lies in $\mathbb{Z}[i]$, and generates a prime ideal in $\mathbb{Z}[i]$. Further $\psi\left(\sigma_{v}\right) \psi^{\tau}\left(\sigma_{v}\right)$ is equal to $p$ (resp. to $p^{2}$ ), when $N v=p$ (resp. $N v=p^{2}$ ), where $v$ divides the rational prime $p$ of $\mathbb{Q}$ and $\tau$ denotes the non-trivial element of $\operatorname{Gal}(L / \mathbb{Q})$.

Let $\chi: G_{\mathbb{Q}} \rightarrow \mathbb{Q}_{\ell}^{*}$ denote the $\ell$-adic cyclotomic character. We have for $p$ coprime to $\ell, \chi\left(\sigma_{p}\right)=p$. Consider the two representations,

$$
\rho_{1}=\chi \oplus \chi \quad \text { and } \quad \rho_{2}=\operatorname{Ind}_{G_{K}}^{G_{\mathbb{Q}}}\left(\psi^{2}\right) .
$$

Let $T$ be the set of rational primes $p$ inert in $L$ and unramified for $\rho_{2}$. The characteristic polynomials of $\rho_{1}\left(\sigma_{p}\right)$ and $\rho_{2}\left(\sigma_{p}\right)$ for $p \in T$, are respectively of the form $(X-p)^{2}$ 
and $X^{2}+p^{2}$. Hence for $i=1,2, \rho_{i}\left(\sigma_{p}\right)^{4}=p^{4} I$, where $I$ is the identity matrix. Thus these representations are potentially equivalent at $T$.

The algebraic monodromy group $G_{1}$ of $\rho_{1}$ is isomorphic to $\mathbb{G}_{m}$. For any natural number $m$, the characters $\psi^{m}$ and $\left(\psi^{\tau}\right)^{m}$ are not equal up to multiplication by a root of unity, by considering the values of the character at places of degree one over $\mathbb{Q}$. It follows that the algebraic monodromy group $G_{2}$ is isomorphic to the normalizer of the Weil restriction of scalars $R_{L / \mathbb{Q}}\left(\mathbb{G}_{m}\right) \subset G L_{2}$. Thus $G_{1}$ is connected, the representations $\rho_{1}$ and $\rho_{2}$ are potentially equivalent at a set of places of density $\frac{1}{2}$, but are not potentially equivalent yielding a contradiction to Theorem 2.1 proved in $[\mathrm{PR}]$.

Example 1.3. Example 1.1 can be generalized. Let $Z \simeq \mathbb{G}_{m}^{k}$ be a split torus of dimension $k$ over a field $E$. The automorphism group of $\mathbb{G}_{m}^{k}$ can be identified with $G L(k, \mathbb{Z})$. Let $\theta \in \operatorname{Aut}(Z)$ be an automorphism of finite order, such that the $\theta$ invariants of $Z$ is a finite group of order $m$. Form the semi-direct product $Z<J>=$ $Z \rtimes \mathbb{Z} / n \mathbb{Z}$, where the generator $1 \in \mathbb{Z} / n \mathbb{Z}$ is denoted by $J$. The multiplication map is defined as, $\left(x J^{k}\right)\left(y J^{l}\right)=x J^{k} y J^{-k} J^{k+l}=x \theta^{k}(y) J^{k+l}$, where $x, y \in Z$. In particular, $J x J^{-1}=\theta(x)$.

Let $\rho$ be an absolutely irreducible representation of $Z<J>$ of dimension greater than 1. The image of $Z$ cannot be trivial, for otherwise, $\rho$ will factor via the group $\mathbb{Z} / n \mathbb{Z}$. Thus $\rho$ cannot be potentially trivial. On the other hand, it can be seen (see 2.4), that the elements of the coset $Z J$ are of order at most $m n$.

Example 1.4. Once there is a pair $\left(\rho_{1}, \rho_{2}\right)$ of representations of $G_{K}$ which fails to be potentially equivalent, then any twist $\left(\rho_{1} \otimes \eta, \rho_{2} \otimes \eta\right)$, where $\eta$ is a finite dimensional linear representation of $G_{K}$, satisfies the hypothesis of being locally potentially equivalent at $T$. Since the character of $\eta$ is non-vanishing in some neighbourhood of identity, the twists are not potentially equivalent either.

Further, if $K \supset K_{0}$, and the representations $\rho_{1} \otimes \eta$ and $\rho_{2} \otimes \eta$ are respectively restrictions to $G_{K}$ of representations $\eta_{1}$ and $\eta_{2}$ of $G_{K_{0}}$, then the pair of representations $\left(\eta_{1}, \eta_{2}\right)$ will also provide a counterexample. These representations will be locally potentially equivalent at the unramified set of places $T_{0}$ of $K_{0}$ that lie below $T$, but will not be potentially equivalent.

1.1. Theorems. Our main theorem says that the failure of $\rho_{1}$ and $\rho_{2}$ being potentially isomorphic stems from the presence of a summand generalizing the above examples, where upto a twist the first representation is trivial and the second representation is abelian.

Given a group $\Gamma$ and a representation $\rho: \Gamma \rightarrow G L(V)$, there is a finite extension $E$ of $F$, over which there is an isotypical decomposition over $E$,

$$
(\rho, V) \simeq \oplus_{i=1}^{t}\left(\rho_{i}, V_{i}\right)
$$

where $\rho_{i}$ are representations of $\Gamma$ to $G L\left(V_{i}\right)$ of the form,

$$
\rho_{i} \simeq r_{i} \otimes \rho_{i}^{\prime} .
$$


Here $r_{i}$ is an absolutely irreducible representation of $\Gamma$, and $\rho_{i}^{\prime}$ is a trivial representation of $\Gamma$ of dimension $n_{i} / \operatorname{dim}\left(r_{i}\right)$, where $n_{i}=\operatorname{dim}\left(V_{i}\right)$.

Theorem 1.1. Let $K$ be a global field and $S$ be a set of places of $K$ containing the archimedean places and of density zero. Let $F$ be a non-archimedean local field of characteristic zero, and $\rho_{1}, \rho_{2}: G_{K} \rightarrow G L_{n}(F)$ be semisimple continuous representations unramified outside $S$, satisfying the following hypothesis:

H1: The algebraic monodromy group $G_{1}$ of $\rho_{1}$ is connected.

H2: There exists a set of places $T$ of $K$ disjoint from $S$ having positive upper density such that the representations $\rho_{1}$ and $\rho_{2}$ are locally potentially equivalent at $T$, i.e., for each $v \in T$, there exists a natural number $m_{v} \geq 1$ such that the $\rho_{1}\left(\sigma_{v}\right)^{m_{v}}$ and $\rho_{2}\left(\sigma_{v}\right)^{m_{v}}$ are conjugate in $G L_{n}(F)$, where $\rho_{1}\left(\sigma_{v}\right)$ and $\rho_{2}\left(\sigma_{v}\right)$ are respectively the Frobenius conjugacy classes at $v$ of $\rho_{1}$ and $\rho_{2}$.

Let $E$ be a finite extension of $F$ such that $\rho_{1}$ has an isotypical decomposition as given by Equations (1.1) and (1.2):

$$
\rho_{1} \simeq \oplus_{i=1}^{t} \rho_{1, i} \simeq \oplus_{i=1}^{t} r_{i} \otimes \rho_{1, i}^{\prime} .
$$

Then there exists a finite extension $L$ of $K$, a set $T_{L}$ of places of $L$ having positive density lying above the set of places $T$ of $K$, and decomposition

$$
\left.\rho_{2}\right|_{G_{L}} \simeq \oplus_{i=1}^{t} \rho_{2, i}
$$

where $\rho_{2, i}$ are semisimple representations of $G_{L}$ to $G L\left(n_{i}, E\right)$ satisfying the following:

(1) For each $i=1, \cdots, t$, there exists a representation $\rho_{2, i}^{\prime}$ of $G_{L}$ into $G L_{n_{i} / \operatorname{dim}\left(r_{i}\right)}(E)$ such that

$$
\left.\rho_{2, i} \simeq r_{i}\right|_{G_{L}} \otimes \rho_{2, i}^{\prime}
$$

(2) The representation $\rho_{2, i}^{\prime}$ factors as a representation $G_{L} \rightarrow Z<J>(E) \rightarrow$ $G L_{n_{i} / \operatorname{dim}\left(r_{i}\right)}(E)$, for some group of the form $Z<J>$ as in Example 1.3.

(3) The trivial representation $\rho_{1, i}^{\prime}$ and $\rho_{2, i}^{\prime}$ of $G_{L}$ are locally potentially equivalent at $T_{L}$.

(4) If either $r_{i}$ or $\rho_{2, i}^{\prime}$ is non-trivial, then the representations $r_{i}$ and $\rho_{2, i}^{\prime}$ are nonisomorphic.

Remark 1.1. In the conclusion of this theorem, a restriction to a finite extension $L$ of $K$ is part of the conclusion. However, it is not possible to remove the phrase 'potential', without sacrificing the positive density of the set of places $T_{L}$, as can be seen from Example 1.2.

Remark 1.2. The decomposition $\rho_{k, i} \simeq r_{i} \otimes \rho_{k, i}^{\prime}$ for $k=1,2$ is not necessarily a motivic decomposition even if $\rho_{i}$ are motivic. Here we consider a representation to be motivic if it satisfies some integrality and purity conditions for the eigenvalues of the Frobenius classes at unramified places.

We now present a different version of the foregoing theorem in terms of character values evaluated at a fixed power of Frobenius classes: 
Theorem 1.2. With notation as in Theorem 1.1, assume that the following hypothesis $\mathbf{H} \mathbf{2}^{\prime}$ is satisfied instead of $\mathbf{H 2}$ :

H2': There exists a set of places $T$ of $K$ disjoint from $S$ having positive upper density, and a natural number $m$ such that for $v \in T$,

$$
\operatorname{Tr}\left(\rho_{1}\left(\sigma_{v}\right)^{m}\right)=\operatorname{Tr}\left(\rho_{2}\left(\sigma_{v}\right)^{m}\right) .
$$

Then the conclusion of Theorem 1.2 remain valid, except that Condition (3) of the conclusion should be replaced by the condition,

$$
\operatorname{Tr}\left(\rho_{1, i}^{\prime}\left(\sigma_{w}\right)^{m}\right)=\operatorname{Tr}\left(\rho_{2, i}^{\prime}\left(\sigma_{w}\right)^{m}\right), \quad \forall w \in T_{L} .
$$

Remark 1.3. Theorem 1.2 differs from Theorem 1.1, in that apart from bounding $m_{v}$ uniformly independent of $v \in T$, we are able to work with character values of the Frobenius classes, rather than the $m$-th powers of the Frobenius classes being conjugate. We don't know whether we can replace $\mathbf{H} \mathbf{2}^{\prime}$ with the following condition:

$$
\operatorname{Tr}\left(\rho_{1}\left(\sigma_{v}\right)^{m_{v}}\right)=\operatorname{Tr}\left(\rho_{2}\left(\sigma_{v}\right)^{m_{v}}\right),
$$

where $m_{v}$ are natural numbers depending on $v$.

1.2. Potential equivalence. The examples given above suggest the possibility of proving potential equivalence of $\rho_{1}$ and $\rho_{2}$ (assuming $G_{1}$ is connected and upper density of $T$ is positive), under some additional natural hypothesis on the nature of the representations $\rho_{1}$ and $\rho_{2}$.

Given a representation $\rho: G_{K} \rightarrow G L_{n}(F)$ as above, consider the commutant algebra for any extension field $E$ of $F$,

$$
C_{K}(\rho, E):=\left\{X \in M_{n}(E) \mid X \rho(g)=\rho(g) X \quad \forall g \in G_{K}\right\} .
$$

When the representation $\rho$ arises is the Galois representation $\rho_{A}$ attached to the Tate module of an abelian variety, Faltings theorem proving Tate's conjecture asserts

$$
C_{K}(\rho, F)=\operatorname{End}_{K}(A) \otimes F .
$$

The algebra $C_{K}(\rho, F)$ is also the commutant of the group $G_{\rho}(F)$ in $M_{n}(F)$. Since the characteristic of $F$ is zero, $C_{K}(\rho, E)=C_{K}(\rho, F) \otimes_{F} E$. For any finite extension $L$ of $K, C_{K}(\rho, F) \subset C_{L}(\rho, F)$. The commutant algebra stabilizes for $L$ sufficiently large: by Zariski density, whenever the image $\rho\left(G_{L}\right)$ is contained inside $G_{\rho}^{0}(F)$, where $G_{\rho}^{0}$ is the connected component of $G_{\rho}$. We call this the stable commutant algebra and denote it by $C_{\bar{K}}(\rho, F)$.

As corollaries of the theorems stated above, we obtain the following theorem providing instances when (global) potential equivalence can be deduced:

Theorem 1.3. With hypothesis as in Theorem 1.2 (or Theorem 1.1), assume further that either of the following conditions hold:

(1) $\rho_{1}$ is absolutely irreducible.

(2) The algebraic monodromy group $G_{2}$ of $\rho_{2}$ is connected. 
(3) The algebraic ranks of $G_{1}$ and $G_{2}^{0}$ are equal, where $G_{2}^{0}$ is the connected component of identity of $G_{2}$.

(4) For any finite extension $L$ of $K$,

$$
C_{K}(\rho, F)=C_{L}(\rho, F)=C_{\bar{K}}(\rho, F) .
$$

Then $\rho_{1}$ and $\rho_{2}$ are potentially equivalent. Further, when $\rho_{1}$ is absolutely irreducible, there exists a character $\chi: G_{K} \rightarrow \bar{F}^{*}$, such that $\rho_{2} \simeq \rho_{1} \otimes \chi$.

The last conclusion is deduced by an argument using Schur's lemma ([Ra]). One cannot expect that the character $\chi$ has values in $F^{*}$ in general, as a pair of representations $\rho$ and $\rho \otimes \chi$, with $\rho$ absolutely irreducible and $\chi$ an arbitrary character with values in $\bar{F}^{*}$ satisfy the hypothesis of the corollary.

Remark 1.4. In general, one can possibly expect the following principle to be valid: suppose a property $P$ of the conjugacy classes of $G L_{n} \times G L_{n}$ is given, such that a pair of $\ell$-adic representations of $G_{K}$ become potentially equivalent whenever the Frobenius classes satisfy property $P$ at a set of places of density one. Then the expectation, barring few exceptions, is that potential equivalence will hold under the weaker assumption that the Frobenius classes satisfy property $P$ at a set of places of positive density, provided one of the algebraic monodromy groups is connected. It is to be hoped that the algebraic method outlined out here can be applied to answer such questions.

1.3. Applications. The above corollary can be applied to the Galois representations attached to modular forms, and we deduce,

Corollary 1.1. Let $f$ and $g$ be two newforms of level $N_{1}$ and $N_{2}$, and respectively of weights $k_{1}$ and $k_{2}$. Suppose that at a set $T$ of primes $p$ coprime to $N_{1} N_{2}$ of positive upper density, and natural numbers $n_{p}, p \in T$,

$$
\operatorname{Tr}\left(T_{p}^{n_{p}}(f)\right)=\operatorname{Tr}\left(T_{p}^{n_{p}}(g)\right),
$$

where $T_{p}$ is the Hecke operator at $p$. Assume further that one of the forms is not CM. Then the weights of the two forms are equal and there exists a Dirichlet character $\chi$ such that for $p \quad \chi N_{1} N_{2}$,

$$
a_{p}(f)=\chi(p) a_{p}(g),
$$

where for a newform $h, a_{p}(h):=\operatorname{Tr}\left(T_{p}(h)\right)$ is the $p$-th Hecke eigenvalue of $h$.

Indeed the corollary follows from either Part (i), (ii) or (ii) of Theorem 1.3, from well known properties of the Galois representation attached to a non-CM newforms.

One of the motivating problems for the questions considered here, is the following application to abelian varieties, which can be deduced from Part (iv) Theorem 1.3. and the theorem of Tate, Zarhin and Faltings proving Tate conjecture on isogenies of abelian varieties:

Corollary 1.2. Let $A, B$ be Abelian varieties of dimension $g$ defined over a number field $K$ without complex multiplication (over $\bar{K}$ ). Suppose that $T$ is a set of finite 
places of positive upper density of $K$ consisting of places of good reduction for $A$ and $B$, such that for every $v \in T$, the reduction $A_{v}, B_{v}$ modulo $v$ of the Abelian varieties $A, B$ at $v$, are isogenous over a finite extension of the residue field $k_{v}$ of $K$ at $v$.

Assume further that the algebraic monodromy group attached to the Galois representation on the $\ell$-adic Tate module of $A$ is connected, and $\operatorname{End}_{K}(B)=\operatorname{End}_{\bar{K}}(B)$, i.e., all the endomorphisms of $B$ are defined over $K$.

Then $A$ and $B$ are isogenous over a finite extension $L$ of $K$.

Remark 1.5. It would be interesting to know whether the statement will hold for abelian varieties in general.

1.4. Outline of the proof. Without any hypothesis on the algebraic monodromy groups, potential equivalence can be deduced by assuming that the density of $T$ is one $([\mathrm{PR}])$. The main thrust of this paper, is to deduce a similar conclusion, assuming only that density of $T$ is positive. The proof of the Theorems 1.1 and 1.2 follow along the lines of ([Se] ) and ([Ra]), by considering the algebraic monodromy groups of the representations involved and converting the problem to one on algebraic groups by means of an algebraic Chebotarev density theorem (see Theorem 2.1). The algebraic Chebotarev density theorem assures the existence of a connected component of the monodromy group of $\rho_{1} \times \rho_{2}$ where the representations are potentially equivalent.

If we assume further that one of the monodromy groups is connected, the algebraic formulation allows us to base change to complex numbers and to employ a version of the unitary trick similar to the one used in [Ra. The crucial and new observation out here is to interpret the consequence of the unitary trick and convert the problem to one involving the first representation and twist of the second representation by an automorphism of finite order (see Equations 2.1, 2.2, 2.3). An appeal to a classical theorem on fixed points of finite order automorphisms of semisimple groups (Theorem 2.4) allows us to get at the algebraic structure of the representations satisfying the hypothesis of Theorem 1.2.

The method of proof allows us to conclude (see Theorem 2.5) that the images of connected components of semisimple algebraic groups with respect to the power maps $P_{m}$ will not collapse. This is unlike the situation given by Examples 1.1 and 1.3, where collapsing happens when the connected component is a torus.

\section{Algebraic formulation of Theorem 1.2}

2.1. A uniform bound for the exponents in Theorem 1.1. We first show that the exponents $m_{v}, v \in T$ appearing in Theorem 1.1 can be uniformly bounded, using the fact that there are only finitely many roots of unity in any non-archimedean local field:

Lemma 2.1. Let $\sigma_{1}$ and $\sigma_{2}$ be two semisimple elements in $G L_{n}(F)$ where $F$ is local field (finite extension of $\mathbb{Q}_{\ell}$ ). Suppose there exists a non-zero integer $k$ such that $\sigma_{1}^{k}$ 
and $\sigma_{1}^{k}$ are conjugate in $G L_{n}(F)$. Then, there exists a positive integer $m$ depending only on $n$ and $F$ such that $\sigma_{1}^{m}$ and $\sigma_{2}^{m}$ are conjugate in $G L_{n}(F)$.

Remark 2.1. Since we are working in $G L_{n}$, two elements are conjugate in $G L_{n}(F)$ if and only if they are conjugate in $G L_{n}(\bar{F})$.

Proof. Choose an algebraic closure $\bar{F}$ of $F$. Let $F^{\prime}$ be the extension of $F$ in $\bar{F}$ generated by the eigenvalues of $\sigma_{1}$ and $\sigma_{2}$ in $\bar{F}$. It is easy to see that

$$
\left[F^{\prime}: F\right] \leq(n !)^{2} \text {. }
$$

The number of roots of unity contained in such a field $F^{\prime}$ is bounded above by some positive integer $m_{0}$ depending only on $\left[F^{\prime}: \mathbb{Q}_{\ell}\right]$, thus depending only on $n$ and $\left[F: \mathbb{Q}_{\ell}\right]$.

Let $\left\{\alpha_{1}, \cdots, \alpha_{n}\right\}$ (respectively $\left\{\beta_{1}, \cdots, \beta_{n}\right\}$ ) be the eigenvalues of $\sigma_{1}$ (respectively $\left.\sigma_{2}\right)$. Since by our hypothesis $\sigma_{1}^{k}$ is conjugate of $\sigma_{2}^{k}$ we have up to a permutation,

$$
\alpha_{i}^{k}=\beta_{i}^{k}, \quad \forall 1 \leq i \leq n .
$$

Hence $\alpha_{i}$ and $\beta_{i}$ differ by a root of unity, which lies in $F^{\prime}$. Thus from the above comment, for $m=m_{0}$ ! we have:

$$
\alpha_{i}^{m}=\beta_{i}^{m}, \quad \forall 1 \leq i \leq n .
$$

But since both $\sigma_{1}$ and $\sigma_{2}$ are semisimple elements in $G L_{n}(\bar{F}), \sigma_{1}^{m}$ and $\sigma_{2}^{m}$ are conjugate in $G L_{n}(F)$.

It follows from this lemma, upon assuming the hypothesis of Theorem 1.1, there exists a positive integer $m$ independent of $v \in T$, and such that for all $v \in T, \rho_{1}\left(\sigma_{v}\right)^{m}$ and $\rho_{2}\left(\sigma_{v}\right)^{m}$ are conjugate in $G L_{n}(F)$.

2.2. An application of an algebraic Chebotarev density theorem. We recall Theorem 3 of [Ra], an algebraic interpretation of results proved in Section 6 (especially Proposition 15) of [Se], giving an algebraic formulation of the Chebotarev density theorem for the density of places satisfying an algebraic conjugacy condition:

Theorem 2.1. [Ra, Theorem 3] Let $M$ be an algebraic group defined over a l-adic local field $F$ of characteristic zero. Suppose

$$
\rho: G_{K} \rightarrow M(F)
$$

is a continuous representation unramified outside a finite set of places of $K$.

Suppose $X$ is a closed subscheme of $M$ defined over $F$ and stable under the adjoint action of $M$ on itself. Let

$$
C:=X(F) \cap \rho\left(G_{K}\right) .
$$

Let $\Sigma_{u}$ denote the set of finite places of $K$ at which $\rho$ is unramified. Then the set

$$
S:=\left\{v \in \Sigma_{u} \mid \rho\left(\sigma_{v}\right) \subset C\right\} .
$$

has a density given by

$$
d(S)=\frac{|\Psi|}{|\Phi|}
$$


where $\Phi$ is the set of connected components of $G$, and $\Psi$ is the set of those $\phi \in \Phi$ such that the corresponding connected component $G^{\phi}$ of $G$ is contained in $X$.

Corollary 2.1. Let $\rho$ be a semisimple continuous $\ell$-adic representation of $G_{K}$ to $G L_{n}(F)$ unramified outside a finite set of places of $K$. Then there is a density one set of places of $K$ at which $\rho$ is unramified and the corresponding Frobenius conjugacy class is semisimple.

Proof. Since the representations are assumed to be semi-simple, the algebraic monodromy groups are reductive algebraic group defined over $F$. The corollary follows from the fact that the semisimple elements in a reductive group $G$ contain a Zariski dense open subset of $G$.

Let $m$ be a natural number. Consider the following Zariski closed, invariant subsets of $G L_{n} \times G L_{n}$ :

$$
\begin{aligned}
X_{m} & :=\left\{\left(g_{1}, g_{2}\right) \in G L_{n} \times G L_{n} \mid \operatorname{Trace}\left(g_{1}^{m}\right)=\operatorname{Trace}\left(g_{2}^{m}\right)\right\} \\
Y_{m} & :=\left\{\left(g_{1}, g_{2}\right) \in G L_{n} \times G L_{n} \mid \operatorname{Trace}\left(\Lambda^{j}\left(g_{1}^{m}\right)\right)=\operatorname{Trace}\left(\Lambda^{j}\left(g_{2}^{m}\right)\right), \quad j=1, \cdots, n\right\},
\end{aligned}
$$

where $\Lambda^{j}$ denotes the $j$-th exterior power representation of $G L_{n}$. For semisimple elements, the condition that $\left(g_{1}, g_{2}\right) \in Y_{m}$, is equivalent to saying that $g_{1}^{m}$ and $g_{2}^{m}$ are conjugate.

Given the hypothesis of Theorem 1.2 (resp. Theorem 1.1), we have

$$
\rho\left(\sigma_{v}\right) \in X_{m}(F) \quad\left(\text { resp. } \rho\left(\sigma_{v}\right) \in Y_{m}(F)\right) \quad \forall v \in T^{\prime},
$$

where $\rho=\rho_{1} \oplus \rho_{2}: G_{K} \rightarrow G L_{n}(F) \times G L_{n}(F)$ is the direct sum of the representations $\rho_{1}$ and $\rho_{2}$. In what follows we present the proof for Theorem [1.2, and make remarks only as required for the proof of Theorem 1.1 .

Let $G$ denote the algebraic monodromy group of $\rho$. Since $T$ is of positive upper density, by Theorem 2.1 above, there exists a connected component $G^{\phi}$ of $G$ such that $G^{\phi}$ is contained in $X_{m}$.

We are led to consider the following problem in the context of algebraic groups: let $G \subset G L_{n} \times G L_{n}$ be a reductive algebraic group and $p_{1}, p_{2}$ denote the two projections. Assume that the image $p_{1}(G)=G_{1}$ is connected. Suppose that there is a connected component $G^{\phi}$ of $G$ contained inside $X_{m}$. What can we conclude about the representations $p_{1}$ and $p_{2}$ of $G$ ? For the rest of this section, we will be following this notation.

We first observe the equivalence of the representations when $G$ is connected:

Proposition 2.2. With the above notation, suppose $G$ is connected and $G \subset X_{m}$. Then the representations $p_{1}$ and $p_{2}$ are equivalent.

Proof. Since $G$ is connected, the $m$-th power map $x \mapsto x^{m}$ from $G$ to $G$ is dominant. Hence $G \subset X_{1}$, and the representations $p_{1}$ and $p_{2}$ are equivalent. 
Remark 2.2. The surjectivity of the $m$-th power map fails when we consider it between connected components. In [PR] the argument continued as follows: it can be assumed that $m$ is chosen such that the $m$-th power map sends $G^{\phi}$ to $G^{0}$, the connected component of identity in $G$. The image will be contained inside the subvariety $X_{1}$. Upon the erroneous assumption that the $m$-th power map is surjective, one concludes that $G^{0} \subset X_{1}$ and this implies potential equivalence of $\rho_{1}$ and $\rho_{2}$. But this assumption is wrong, as was pointed out by J.-P. Serre.

2.3. A unitary trick. One of the advantages with the algebraic formulation is that it allows base change to the field of complex numbers, which makes it amenable to transcendental methods. Choosing an isomorphism of the algebraic closure of $F$ with $\mathbb{C}$, we consider the analogous problem over complex numbers.

Let $U$ be a maximal compact subgroup of $G(\mathbb{C})$ which we will assume is contained inside $U(n) \times U(n)$, where $U(n) \subset G L_{n}(\mathbb{C})$ is the (standard) group of unitary $n \times n$ matrices. The group $U$ is Zariski dense in $G$. Hence the intersection $U^{\phi}:=U \cap G^{\phi}$ is Zariski dense in $G^{\phi}$. In particular, it is non-empty.

The image $p_{1}(U)$ of the projection of $U$ to the first factor is a maximal compact subgroup of $G_{1}(\mathbb{C})$. Since by hypothesis $G_{1}$ is connected, $p_{1}(U)$ is connected and equal to $p_{1}\left(U^{0}\right)$, where $U^{0}:=U \cap G^{0}$ is the connected component of $U$. Hence for any connected component $U^{\psi}$ of $U, p_{1}\left(U^{\psi}\right)=p_{1}\left(U^{0}\right)$. In particular, the image $p_{1}\left(U^{\phi}\right)$ is a subgroup of $G_{1}(\mathbb{C})$.

Hence there exists an element of the form $\left(I_{n}, j\right) \in U^{\phi}$, where $I_{n}$ denotes the identity matrix in $G L_{n}(\mathbb{C})$. Since $G^{\phi} \subset X_{m}$,

$$
n=\operatorname{Tr}\left(I_{n}^{m}\right)=\operatorname{Tr}\left(j^{m}\right) .
$$

Since the only unitary matrix in with trace equal to $n$ is the identity matrix, we conclude that $j^{m}=I_{n}$. Thus, $G^{\phi}=G^{0}\left(I_{n}, j\right)$ with $j^{m}=I_{n}$. Let $J=\left(I_{n}, j\right)$. The condition $G^{\phi} \subset X_{m}$, translates to the condition $(x J)^{m} \in X_{m}$ for any $x \in G^{0}$.

Remark 2.3. In Ra, we considered the case $m=1$. In this case, we have that $\left(I_{n}, I_{n}\right) \in G^{\phi}(\mathbb{C})$, and this implies that $G^{\phi}=G^{0}$, and the representations $p_{1}$ and $p_{2}$ are isomorphic restricted to $G^{0}$. Hence we conclude that $\rho_{1}$ and $\rho_{2}$ are potentially isomorphic.

For $m \geq 2$, the significance of the unitary trick lies in the following crucial observation:

$$
(x J)^{m}=x J \cdots x J=x J x J^{-1} J^{2} x J^{-2} \cdots J^{m-1} x J^{-(m-1)} J^{m-1} J, \quad x \in G^{0} .
$$

Let $\theta(x)=J x J^{-1}$ denote the automorphism of finite order (dividing $m$ ) of $G^{0}$ induced by the conjugation action of $J$. Since by the unitary trick $J^{m}=\left(I_{n}, I_{n}\right)$, the above equation becomes,

$$
(x J)^{m}=x \theta(x) \theta^{2}(x) \cdots \theta^{m-1}(x), \quad x \in G^{0} .
$$


The condition $G^{\phi} \subset X_{m}$ now translates to the following the first representation is absolutely irreducible condition on the representations $p_{1}$ and $p_{2}$ of $G^{0}$ :

$$
\operatorname{Tr}\left(p_{1}(x)^{m}\right)=\operatorname{Tr}\left(p_{2}\left(x \theta(x) \theta^{2}(x) \cdots \theta^{m-1}(x)\right)\right), \quad x \in G^{0} .
$$

2.4. Example 1.3. The above calculation can be reversed. We put ourselves in the context of Example 1.3. For $x \in Z$,

$$
(x J)^{n}=x \theta(x) \cdots \theta^{n-1}(x),
$$

is $\theta$ invariant as $Z$ is abelian. Since, by assumption the subgroup of $\theta$-invariants of $Z$ is finite (of order $m$ ), the element $x J$ is of finite order. Hence for any representation $\rho$ of $Z\langle J\rangle$, the elements of the coset $x J$ have order at most $m n$, where $m$ is the order of the subgroup of $\theta$-invariants of $Z$.

Remark 2.4. We make a remark, which we will use in deducing Part (iv) of Theorem 1.3. If $\rho(Z)$ and $\rho(J)$ commute, the fact that $x J$ is of finite order implies that $\rho(x)$ is of finite order for $x \in Z$. Since $Z$ is connected, its image is trivial and this contradicts the irreducibility of $\rho$. In particular, $\rho(Z)$ and $\rho(J)$ do not commute, i.e., there exists elements of $\rho(Z)$ which are not fixed by the conjugacy action of $\rho(J)$.

2.5. Algebraic analogue of Theorem 1.2. In this section, we consider the subgroup of $G \subset G L(n) \times G L(n)$ generated by $G^{0}$ and the the connected component $G^{\phi}$, as considered above. By an abuse of notation, we continue to denote this subgroup by $G$. Since $J$ has finite order, it is semisimple with eigenvalues roots of unity. Hence $J$ and the automorphism $\theta$ are defined over a finite extension $F^{\prime}$ of $F$. The group $G$ is defined over $F$, and over $F^{\prime}$, is isomorphic to the group generated by $G^{0}$ and the element $J=(1, j) \in G\left(F^{\prime}\right)$. The conjugation action by the element $(1, j)$ induces the automorphism $\theta$ on $G^{0}$ defined over $F^{\prime}$. We decompose $G$ (the decompositions are valid over any finite extension of $F$ containing $F^{\prime}$ ) with respect to the action of $\theta$ as follows:

(1) The derived subgroup $G^{\prime}$ of $G^{0}$ is a connected semisimple group defined over $F$.

(2) The connected component $Z$ of the center of $G^{0}$ is invariant under $\theta$.

The automorphism $\theta$ leaves stable the groups $G^{\prime}$ and $Z$ (considered as subgroups over $F^{\prime}$ ), and there is a decomposition

$$
G^{0}=G^{\prime} Z \text { and } G^{\prime} \cap Z \text { is finite. }
$$

We further decompose $Z$ with respect to the action of $\theta$.

Consider the lattice $X^{*}(Z)$ of characters of $Z$. Define endomorphisms of $X^{*}(Z)$,

$$
I_{\theta}=1-\theta \quad \text { and } \quad N_{\theta}=1+\theta+\cdots+\theta^{m-1} .
$$

Let $X^{\theta}$ be the subgroup of $X^{*}(Z)$ on which $\theta$ acts trivially and $Z^{\theta}$ be the corresponding subtorus of $Z$ defined over $F^{\prime}$. This is the maximial subtorus of $Z$ on which $\theta$ acts trivially. 
(3) Decompose the space $X^{*}(Z) \otimes \mathbb{Q}=X^{\theta} \otimes \mathbb{Q} \oplus Y$, where $Y$ is the kernel of $N_{\theta}$. Choose a lattice $L_{0} \subset Y$ and let $L_{\theta}=\sum_{i=0}^{m-1} \theta^{i}\left(L_{0}\right)$. The lattice $L_{\theta}$ is $\theta$-invariant and is the character group of a $\theta$-stable subtorus $Z_{\theta}$ of $Z$. The invariants of $\theta$ acting on $Z_{\theta}$ and $G^{\prime} Z^{\theta} \cap Z_{\theta}$ are finite groups.

(4) Let $Z_{\theta}<J>$ be the subgroup of $G$ generated by $Z_{\theta}$ and the element $J$. The groups $G^{\prime} Z^{\theta}$ and $Z_{\theta}<J>$ are normal in $G$, and there is a decomposition

$$
G=G^{\prime} Z^{\theta}\left(Z_{\theta}<J>\right) \text { and } G^{\prime} Z^{\theta} \cap Z_{\theta}<J>\text { is finite. }
$$

We have the following algebraic analogue of Theorem 1.2

Theorem 2.3. Let $G$ be as above. Suppose that the following condition is satisfied for $x \in G^{0}$ :

$$
\operatorname{Tr}\left(p_{1}\left(x^{m}\right)\right)=\operatorname{Tr}\left(p_{2}\left(x \theta(x) \cdots \theta^{m-1}(x)\right)\right) .
$$

With respect to the decomposition $G=G^{\prime} Z^{\theta} Z_{\theta}<J>$ given above, the following hold:

(1) $\theta$ acts trivially on $G^{\prime} Z^{\theta}$, and

$$
\left.\left.p_{1}\right|_{G^{\prime} Z^{\theta}} \simeq p_{2}\right|_{G^{\prime} Z^{\theta}}
$$

(2) $p_{1}$ restricted to $Z_{\theta}<J>$ is trivial.

(3) Let $E$ be a finite extension of $F^{\prime}$, over which the representation $p_{1}$ of $G$ has an isotypical decomposition as given by Equations (1.1) and (1.2):

$$
p_{1} \simeq \oplus_{i=1}^{t} p_{1, i} \simeq \oplus_{i=1}^{t} R_{i} \otimes p_{1, i}^{\prime}
$$

where the representations $p_{1, i}^{\prime}$ are trivial of appropriate degree. The representation $p_{2}$ decomposes over $E$ as

$$
p_{2} \simeq \oplus_{i=1}^{t} p_{2, i}
$$

where $p_{2, i}$ are representations of $G$ to $G L_{V_{i}}$. For $1 \leq i \leq t$, there exists representations $p_{2, i}^{\prime}$ of $Z_{\theta}<J>$ into $G L_{n_{i} / \operatorname{dim}\left(R_{i}\right)}(E)$, satisfying:

(a) As representations of the group $G^{\prime} Z^{\theta} \times Z_{\theta}<J>$,

$$
p_{2, i}=R_{i} \otimes p_{2, i}^{\prime} .
$$

where $R_{i}$ is considered as a representation of $G^{\prime} Z^{\theta}$.

(b) The representations $p_{1, i}^{\prime}, p_{2, i}^{\prime}$ satisfy,

$$
n_{i}=\operatorname{Tr}\left(p_{1, i}^{\prime}\left(x^{m}\right)\right)=\operatorname{Tr}\left(p_{2, i}^{\prime}\left(x \theta(x) \cdots \theta^{m-1}(x)\right)\right), \quad x \in Z_{\theta} .
$$

Equivalently, for $y=x J$ belonging to the coset $Z_{\theta} J$,

$$
n_{i}=\operatorname{Tr}\left(p_{1, i}^{\prime}\left(y^{m}\right)\right)=\operatorname{Tr}\left(p_{2, i}^{\prime}\left(y^{m}\right)\right) .
$$

Remark 2.5. A similar analogous statement can be made for Theorem 1.1, where the hypothesis is modified by considering the analogue of Equation (2.4) for all exterior powers. Similarly the conclusion can be strengthened to say that the equality of Equation (2.5) holds for all exterior powers. Since $Z_{\theta}$ is a torus, the elements in the image of $Z_{\theta}<J>$ will be semisimple for any linear representation of $Z_{\theta}<J>$. From this and the equality of traces for all exterior powers, one concludes that the elements $p_{1, i}^{\prime}\left(y^{m}\right)$ and $p_{2, i}^{\prime}\left(y^{m}\right)$ are conjugate for $y=x J \in Z_{\theta} J, x \in Z_{\theta}$. 
2.6. Proof of Parts (1) and (2) of Theorem 2.3. Let $H$ denote the connected component of identity of $\operatorname{Ker}\left(\left.p_{1}\right|_{G^{\prime}}\right)$. The group $H$ is semisimple, and since $p_{1}=p_{1} \circ \theta$, $H$ is $\theta$-stable. Let $H^{\theta}$ denote the identity connected component of the fixed points of $\theta$ acting on $H$.

Restricted to $H^{\theta}$, Equation (2.4) gives the identity:

$$
n=\operatorname{Tr}\left(p_{1}\left(x^{m}\right)\right)=\operatorname{Tr}\left(p_{2}\left(x^{m}\right)\right), \quad x \in H^{\theta},
$$

where we have used the fact that $p_{1}$ restricted to $H$ is trivial. By Proposition 2.2, the representation $p_{2}$ restricted to $H^{\theta}$ is trivial. Since $G^{\prime} \subset G L_{n} \times G L_{n}$, the representation $p_{2}$ restricted to $H$, and in particular to $H^{\theta}$ is injective. Hence $H^{\theta}$ is trivial.

The following theorem is classical (see for instance [K, Chapter 8]) or [BM]), and we give a proof in our context in the following section):

Theorem 2.4. Let $H$ be a (non-trivial) connected semisimple group over $F$, and let $\theta$ be a finite order automorphism of $H$. Then the connected component $H^{\theta}$ of the fixed points of $\theta$ is a non-trivial reductive group.

From this theorem and the triviality of $H^{\theta}$, it follows that $H$ is trivial, i.e., the kernel of $\left.p_{1}\right|_{G^{\prime}}$ is finite. From the equality $p_{1} \circ \theta=p_{1}$, we get for $g \in G^{\prime}$ that $\theta(g)=z g$ for some $z$ in kernel of $p_{1}$, i.e., $\theta(g) g^{-1}=z$. The finiteness of the kernel and the connectedness of $G^{\prime}$ implies that $\theta$ acts trivially on $G^{\prime}$.

Thus $\theta$ acts trivially on $G^{\prime} Z^{\theta}$. Proposition 2.2 applied to Equation (2.4) restricted to $G^{\prime} Z^{\theta}$ yields Part (1) of Theorem 2.3,

The proof of Part (2) of Theorem 2.3, follows from the fact that $\theta$ acts trivially on the first co-ordinate. Since $Z_{\theta}$ has finite $\theta$-invariants, being connected, it cannot have a non-trivial quotient with trivial action of $\theta$. The element $J$ projects trivially to the first component by our choice of $J$.

2.7. Part (3) of Theorem 2.3. By Part (1), after a change of basis of one of the representations we can assume that

$$
\left.p_{1}\right|_{G^{\prime} Z^{\theta}}=\left.p_{2}\right|_{G^{\prime} Z^{\theta}}
$$

Let $E^{n}=\bigoplus_{i=1}^{t} V_{i}$ be the decomposition into isotypical components of absolutely irreducible representations of the representation $\left.p_{1}\right|_{G^{\prime} Z^{\theta}}$. These components are stabilized by $G$ as $G^{\prime} Z^{\theta}$ is normal in $G$. Hence, considered as representations of the group $G^{\prime} Z^{\theta} \times Z_{\theta}<J>$, the representation $p_{2, i}$ can be written as,

$$
p_{2, i} \simeq \oplus_{j=1}^{n_{j}} R_{i} \otimes \eta_{i j}
$$

for some collection of absolutely irreducible representations $\eta_{i j}$ of $Z_{\theta}<J>$. Define

$$
p_{2, i}^{\prime}=\oplus_{j=1}^{n_{j}} \eta_{i j}
$$

This gives the decomposition in Part (3.a). 
To prove Part (3.b), in terms of the decompositions, Equation (2.4) can be written as,

$$
\sum_{i=1}^{t} \operatorname{Tr}\left(R_{i}\left(g^{m}\right)\right) \operatorname{Tr}\left(p_{1, i}^{\prime}\left(x^{m}\right)\right)=\sum_{i=1}^{t} \operatorname{Tr}\left(R_{i}\left(g^{m}\right)\right) \operatorname{Tr}\left(p_{2, i}^{\prime}\left(x \theta(x) \cdots \theta^{m-1}(x)\right)\right),
$$

for $g \in G^{\prime} Z^{\theta}$ and $x \in Z_{\theta}$. Since $G^{\prime} Z^{\theta}$ is connected, the map $g \mapsto g^{m}$ is surjective. The representations $R_{i}$ are distinct irreducible representations of $G^{\prime} Z^{\theta}$. Hence by the linear independence of characters, we obtain Part (3.c) of Theorem 2.3 .

2.8. Proof of Theorem 2.4. For the sake of completeness of exposition, we give a proof of Theorem 2.4 in the following context which is sufficient for our purpose (see [K, Chapter 8] for a more detailed and complete exposition): we take $F=\mathbb{C}$, $G \subset G L(n, \mathbb{C})$ and the automorphism $\theta$ is induced by conjugation by an unitary matrix. In this case, $\theta$ commutes with the Cartan involution $\theta_{c}: A \mapsto^{t} \bar{A}^{-1}$. Hence the Cartain involution fixes $H^{\theta}$, and it follows that $H^{\theta}$ is a reductive group.

To see that the connected component $H^{\theta}$ of the fixed points of $\theta$ is non-trivial, it suffices to work with a compact form of $H$. Let $\mathfrak{H}$ denote the complexification of the Lie algebra of the compact form. We continue to denote by $\theta$ the inducted action on the Lie algebra. Decompose $\mathfrak{H}=\bigoplus_{i=0}^{m-1} \mathfrak{H}_{i}$ as eigenspaces for the action of $\theta$ on $\mathfrak{H}$, where $\mathfrak{H}_{j}=\left\{X \in \mathfrak{H} \mid \theta(X)=\zeta_{m}^{j} X\right\}$. Here $\zeta_{m}$ is a fixed choice of a primitive $m$-th root of unity.

Let $I$ be the set of indices for which $\mathfrak{H}_{i}$ is non-zero, and let $k \in I$ be such that $\zeta_{m}^{k}$ is a generator for the subgroup of the roots of unity generated by $\zeta_{m}^{j}, j \in I$. For $X \in \mathfrak{H}_{k}$ and $Y \in \mathfrak{H}_{l}$,

$$
\theta(\operatorname{ad}(X)(Y))=\theta([X, Y])=[\theta(X), \theta(Y)]=\zeta_{m}^{k+l}[X, Y]
$$

Suppose 0 is not in $I$. For any $n, a d(X)^{n}(Y)$ belongs to $\zeta_{m}^{n k+l}$-eigenspace of $\theta$. The assumption on $k$ implies that for some $n, \operatorname{ad}(X)^{n}$ will annihilate the space $\mathfrak{H}_{l}$. Choosing $n$ appropriately, this implies that $\operatorname{ad}(X)$ is nilpotent on $\mathfrak{H}$. But then there are no such elements in $\mathfrak{H}$. Hence $0 \in I$ and this implies that $H^{\theta}$ is non-trivial.

2.9. Images of connected components with respect to power maps. Examples 1.1 and 1.3 gives instances when the power map $P_{m}: x \mapsto x^{m}$ from a connected component $G^{\phi}$ of $G$ to the connected component of identity has image a singleton set. Such instances occur when the connected component is a tori. The observation given by Equation (2.1) allows us to conclude that the images of connected components of semisimple algebraic groups with respect to the power maps $P_{m}$ will not collapse.

Theorem 2.5. Let $G$ be a semisimple algebraic group over an algebraically closed field $F$ of characteristic zero. Let $G^{\phi}$ denote a connected component of $G$. Suppose that the order of $G^{\phi}$ is $n$. For a sufficiently large multiple $m$ of $n$, the image of $G^{\phi}$ by the power map $P_{m}: x \mapsto x^{m}$ contains a (Zariski open) neighbourhood of identity of a non-trivial connected reductive group. 
Proof. We show that there exists a torsion element $j$ in $G^{\phi}$ of order $m$. Elements $x$ of $G^{\phi}$ induce automorphisms $\theta_{x}$ of $G^{0}$ by conjugation, and two such automorphisms are related by an inner automorphism. By $[\mathrm{Sp}$, Corollary 2.14] the sequence

$$
1 \rightarrow \operatorname{Inn}\left(G^{0}\right) \rightarrow \operatorname{Aut}\left(G^{0}\right) \rightarrow \operatorname{Out}\left(G^{0}\right) \rightarrow 1,
$$

splits. Since $\operatorname{Out}\left(G^{0}\right)$ is finite, modifying $x$ by an element in $G^{0}$, we get an element $j \in G^{\phi}$, such that the corresponding inner automorphism it defines on $G^{0}$ is trivial. Hence $j$ belongs to the center of $G^{0}$. Since the center of $G^{0}$ is finite, this yields an element $j \in G^{\phi}$ of finite order.

Let $\theta_{j}(x)=j x j^{-1}$ be the automorphism induced on $G^{0}$ by conjugation by $j$. From Equation (2.2), the image of $P_{m}$ will contain elements of the form $x \theta(x) \theta^{2}(x) \cdots \theta^{m-1}(x)$, where $x$ ranges over the elements of $G^{0}$. In particular, the image will contain elements of the form $x^{m}$ for $x \in\left(G^{0}\right)^{\theta}$, where $\left(G^{0}\right)^{\theta}$ denotes the connected component of identity of the fixed points of $\theta$ acting on $G^{0}$. By Theorem 2.4, this is a non-trivial connected reductive group. The image of the map $x \mapsto x^{m}$ from $\left(G^{0}\right)^{\theta}$ to itself is dominant and contains a neighbourhood of identity of $\left(G^{0}\right)^{\theta}$, and this proves the theorem.

\section{Proof of Theorem 1.2}

3.1. Algebraic envelopes. We first make some few remarks on the relationship between representations of abstract groups and their algebraic envelopes:

(1) Suppose $\Gamma \subset G(\bar{F})$ is a Zariski dense subgroup and $\rho$ a representation of $G$. Then the isotypical decompositions $\left.\rho\right|_{\Gamma}$ is the restriction to $\Gamma$ of the isotypical decomposition of $\rho$. This follows from the fact that a representation $R$ of $G$ is irreducible if and only if it is irreducible restricted to $\Gamma$.

(2) Suppose $\rho: \Gamma \rightarrow G L_{n}(\bar{F})$ is a representation with connected algebraic monodromy group $G$. If $r: G \rightarrow H$ is a representation of algebraic groups, then the algebraic envelope of $r \circ \rho$ is the image group $r(G)$, and hence is also a connected group. In particular if $\rho \simeq \bigoplus_{i=1}^{t} \rho_{i}$, then the algebraic envelopes of $\rho_{i}$ are connected.

(3) Suppose $\rho_{1}, \rho_{2}$ are two linear representations of $\Gamma$. The tensor product representation 'factors' via the direct sum: $\gamma \mapsto\left(\rho_{1}(\gamma), \rho_{2}(\gamma)\right) \mapsto \rho_{1}(\gamma) \otimes \rho_{2}(\gamma)$. Further $\rho_{1}(\gamma) \otimes \rho_{2}(\gamma)$ is trivial if and only if both $\rho_{1}(\gamma)$ and $\rho_{2}(\gamma)$ are trivial. Hence the algebraic monodromy group of $\rho_{1} \otimes \rho_{2}$ and $\rho_{1} \oplus \rho_{2}$ are isomorphic.

3.2. Proof of Theorem 1.2. We deduce now Theorem 1.2 from Theorem 2.3, From the arguments of Sections 2.2 and 2.3, consider the group $\tilde{G}$ generated by $G^{0}$ and the connected component $G^{\phi}$ (as is done in Section 2.5). The group $\rho^{-1}(\tilde{G}(F)$ ) is of finite index in $G_{K}$ and is of the form $G_{K_{1}}$ for some finite extension $K_{1}$ of $K$. We apply Theorem 2.3 to $\tilde{G}$ and let $E$ be the field given in Part (3) of Theorem 2.3. 
The natural map $\pi$ from the product of groups

$$
\pi: \tilde{G}^{\prime} Z^{\theta}(E) \times Z_{\theta}<J>(E) \rightarrow \tilde{G}(E)
$$

has finite kernel $A$. Choose a $\theta$-stable open subgroup $M^{\prime}$ (resp. $M_{\theta}$ ) of $\tilde{G}^{\prime} Z^{\theta}(E)$ (resp. $Z_{\theta}<J>(E)$ ), such that $M^{\prime} \times M_{\theta}$ intersects $A$ only at the identity element. Let $M_{\theta}<J>$ denote the open subgroup of $Z_{\theta}<J>(E)$ generated by $M_{\theta}$ and $J$. This group is Zariski dense in $Z_{\theta}<J>$.

The group $M^{\prime} \times M_{\theta}<J>$ maps isomorphically via $\pi$ to its image group denoted by $M$ in $\tilde{G}(E)$. This is an open subgroup of $\tilde{G}(E)$. The intersection $\rho\left(G_{K_{1}}\right) \cap M$ is of finite index in $\rho\left(G_{K_{1}}\right)$, and is of the form $\rho\left(G_{L}\right)$ for some finite extension $L$ of $K$. Thus we can consider the map $\rho: G_{L} \rightarrow \tilde{G}(E)$ to factor via $\tilde{G}^{\prime} Z^{\theta}(E) \times Z_{\theta}<J>$ $(E)$. Composing the maps $R_{i}, p_{1, i}^{\prime}$ and $p_{2, i}^{\prime}$ with the map $\rho$ defines respectively linear representations $r_{i}, \rho_{1, i}^{\prime}$ and $\rho_{2, i}^{\prime}$ of $G_{L}$.

By the above remark, the isotypical decomposition of the representation $\rho_{1}$ restricted to $G_{L}$ is the restriction of the isotypical decomposition of $\rho_{1}$. Since the representations $R_{i}$ and $p_{2, i}^{\prime}$ factor via different groups, provided one of them is non-trivial, they are not isomorphic.

We need to only check the positive density upon restriction to $L$. From the construction, the algebraic monodromy of the collection of representations $\rho_{2, i}^{\prime}$ is $Z_{\theta}<J>$. The set of places $T_{L}$ is defined to be those places of $L$ such that the image of the Frobenius conjugacy class by the representations $\rho_{2, i}^{\prime}$ lands inside the component $Z_{\theta} J$ of $Z_{\theta}<J>$, and hence it is of positive density by Chebotarev density theorem. Theorem 1.2 follows now from Theorem 2.3 .

\section{Proof of Theorem 1.3}

We now prove Theorem 1.3 .

(1) If $\rho_{1}$ is absolutely irreducible, then $t=1$ and the dimension of the representations $\rho_{1,1}^{\prime}$ and $\rho_{2,1}^{\prime}$ is one. Since they are also potentially equivalent, the values of $\rho_{2,1}^{\prime}$ lie in roots of unity of $F$, which is a finite set. Hence the representations are potentially equivalent.

(2) Suppose that the algebraic monodromy group of $\rho_{2}$ is also connected. By Remark 3.1, the algebraic monodromy groups of the components $\rho_{2, i} \simeq r_{i} \otimes \rho_{2, i}^{\prime}$ are also connected for each $i$. Further the algebraic monodromy group of the tensor product $r_{i} \otimes \rho_{2, i}^{\prime}$ and $r_{i} \oplus \rho_{2, i}^{\prime}$ are isomorphic. This implies that the algebraic monodromy group of $\rho_{2, i}^{\prime}$ are also connected.

Thus we are in the situation of the hypothesis of Theorem 1.2, where the first representation $\rho_{1, i}^{\prime}$ is trivial and the second representation $\rho_{2, i}^{\prime}$ has connected algebraic monodromy group. In this case, the algebraic monodromy group of $\rho_{1, i}^{\prime} \oplus \rho_{2, i}^{\prime}$ is isomorphic to that of $\rho_{2, i}^{\prime}$, and hence is connected. By Proposition 2.2, Part (2) of Theorem 1.3 follows. 
(3) The projection map takes a maximal torus in the algebraic monodromy group of $\rho_{2}$ to a maximal torus of the algebraic monodromy group of $r_{i} \otimes \rho_{2, i}^{\prime}$ for each $i$. The latter monodromy group is isomorphic to the monodromy of $r_{i} \oplus \rho_{2, i}^{\prime}$. If the ranks of the monodromy groups of $\rho_{1}$ and $\rho_{2}$ are equal, then for each $i$, the representations $\rho_{2, i}^{\prime}$ are finite. This means that the representations $\rho_{2, i}^{\prime}$ are potentially trivial and hence the representations $\rho_{1}$ and $\rho_{2}$ are potentially equivalent.

(4) The representations $\rho_{2, i}^{\prime}$ factors via the group $Z_{\theta}<J>(E)$. By Remark 2.4, if $\rho_{2, i}^{\prime}$ has an irreducible component of dimension at least two for some $i$, then there exists an element, say $u \in Z_{\theta}(E)$, such that $\rho_{2, i}^{\prime}(u)$ is not invariant by the conjugacy action induced by $J$. Since $Z$ is abelian, this means that the stable commutant algebra of $\rho_{2, i}^{\prime}$ is strictly larger than the the commutant algebra of $\rho_{2, i}^{\prime}$. This implies that $C_{K}\left(\rho_{2}, F\right) \neq C_{\bar{K}}\left(\rho_{2}, F\right)$.

Hence if the commutant algebra $C_{K}\left(\rho_{2}, F\right)$ is isomorphic to the stable commutant algebra $C_{\bar{K}}\left(\rho_{2}, F\right)$, the representations $\rho_{2, i}^{\prime}$ breaks up as a direct sum of one dimensional representations over $\bar{E}$. In particular $p_{2, i}^{\prime}\left(Z_{\theta}\right)$ and $p_{2, i}^{\prime}(J)$ commute, where $p_{2, i}^{\prime}$ is the representation of ${ }_{\theta}<J>$ through which $\rho_{2, i}^{\prime}$ factors. But as remarked in Section 2.4, this implies that the representations $p_{2, i}^{\prime}$ is finite, and hence $\rho_{2, i}^{\prime}$ is potentially finite.

This proves Theorem 1.3 .

Acknowledgement. Our sincere thanks to J.-P. Serre for pointing out an error in our earlier paper $([\mathrm{PR}])$, and for his continued interest which motivated us to persist with this question. We thank the referee for useful suggestions.

The first author thanks the School of Mathematics, Tata Institute of Fundamental Research for excellent work environment and hospitality. The first author also thanks Mathematical Research Impact Centric Support (MATRICS) grant under Science and Engineering Research Board, Department of Science and Technology, Government of India for supporting his travels that helped with this collaboration.

\section{REFERENCES}

[BM] A. Borel and G. D. Mostow, On Semi-Simple Automorphisms of Lie Algebras, Annals of Mathematics Second Series, Vol. 61, No. 3 (May, 1955), pp. 389-405.

[K] V. G. Kac, Infinite dimensional Lie algebras, Third edition. Cambridge University Press, Cambridge, 1990. xxii+400 pp.

[Ko] E. Kowalski, Some local-global applications of Kummer theory, Manuscripta Math. 111 (2003) 105-139.

[KPR] M. Kulkarni, V. M. Patankar and C. S. Rajan, Locally potentially equivalent two dimensional Galois representations and Frobenius fields of elliptic curves, J. Number Theory, 164 (2016) 87-102.

[PR] Vijay M. Patankar and C. S. Rajan, Locally potentially equivalent Galois representations, J. of Ramanujan Math. Soc., Vol. 27, No. 1, (2012), 77-90.

[Ra] C. S. Rajan, On strong multiplicity one for l-adic representations, International Math. Res. Notices, 1998, No.3, pp. 161-172. 
[Se] J.-P. Serre, Quelques applications du théorème de densité de Chebotarev, Inst. Hautes Études Sc. Publ. Math. 54 (1981) 323-4-1.

[Sp] T. A. Springer, Reductive Groups, in Automorphic forms, representations and L-functions (Proc. Sympos. Pure Math., Oregon State Univ., Corvallis, Ore., 1977), Part 1, pp. 3-27, Proc. Sympos. Pure Math., XXXIII, Amer. Math. Soc., Providence, R.I., 1979.

Department of Mathematics, Bits Pilani, Goa campus, Zuarinagar, Goa, India 403726., EMAIL: VIJAYPATANKAR@GMAIL.COM

School of Mathematics, Tata Institute of Fundamental Research, Dr. Homi Bhabha Road, Colaba, Bombay, INDIA 400005., EMAIL: RAJAN@Math.TiFr.REs.in 